\title{
Shortest Paths and Loop-Free Routing in dynamic networks (Extended Abstract)
}

\author{
Baruch Awerbuch * \\ Dept. of Mathematics and Lab. for Computer Science, \\ M.I.T., \\ Cambridge, MA 02139 \\ ARPANET: baruch@theory.lcs.mit.edu
}

\begin{abstract}
In this paper, we survey the existing methods for designing shortest paths routing algorithms for dynamic networks. We compare them based on worstcase communication and message complexity, and suggest new approach that yields a protocol with linear time and polynomial communication.

The main idea behind our approach is to use a "dynamic synchronizer", which transforms a dynamic asynchronous network into static synchronous one. We believe this is an important methodology in design and analysis of communication protocols, that can be applied to other problems as well.
\end{abstract}

\section{Introduction}

\subsection{Motivation}

The Dynamic Asynchronous network, where links may repeatedly fail and recover, is a realistic model of existing commercial communication networks, such as the ARPANET [MRR80]. Design and analysis of protocols for such networks is much more complicated than the corresponding tasks in a Static

"Supported by Air Force Contract TNDGAFOSR-860078, ARO contract DAAL03-86-K-0171, NSF contract CCR8611442, and a special grant from IBM.

Permission to copy without fee all or part of this material is granted provided that the copies are not made or distributed for direct commercial advantage, the ACM copyright notice and the title of the publication and its date appear, and notice is given that copying is by permission of the Association for Computing Machinery. To copy otherwise, or to republish, requires a fee and/or specific permission.

- 1990 ACM 089791-405-8/90/0009/0177...\$1.50
Synchronous network. Unfortunately, the latter model is not a realistic model of a communication network. Dynamic networks have long been thought to be a much less efficient and a much more complex environment then static networks. This common belief has been due to the existence of a large class of problems which could be solved much faster in static networks than in dynamic networks.

One example of such problem is shortest paths and loop-free routing. Those tasks can be efficiently implemented by trivial algorithms in static synchronous network. This paper argues that the right approach to construct shortest paths in dynamic networks and to achieve loop-free routing is to reduce those problems to analogous problems in static synchronous network. This achieves the best known dynamic shortest paths algorithm, which requires linear time and polynomial communication.

\subsection{Models and Complexity Mea- sures}

The purpose of this sub-section is to clarify the models and to establish common grounds in order to evaluate performance of different protocols in different network models.

\subsubsection{Static synchronous network}

The communication network is described by a fixed graph $G(V, E)$; the delay of each link is exactly one time unit of the global clock, accessible to all the nodes. In the rest of the paper, we denote $|V|=n$. We also denote by $D$ the diameter of $G$.

Only "short" messages (say, containing at most $\log n$ bits) are allowed; longer messages should be chopped in small pieces. At most one message can be sent per link per pulse. 
Complexity measures are defined as follows: the communication complexity of a protocol is the number messages sent by the protocol, and the time complexity is the number of pulses it takes the protocol from its start until completion.

\subsubsection{Static asynchronous network}

The communication network is again described by a fixed graph $G(V, E)$; the delay of each link is bounded but there is no global clock. That is, communication is reliable but completely asynchronous.

The only difference in complexity measures is the way the time complexity is defined. We use the following definition, first suggested by Gallager [Gal82]. Given an execution of a protocol in an asynchronous network, normalized time of the execution is the time measured by some global clock, assuming that the delay of each link varies arbitrarily between 0 and 1 . In order to account for link congestion, this assumption is made only for message at the front of the link queue, i.e. transmitting 1000 messages over the same link may take 1000 units of time. We define worst-case time complexity of a protocol to be maximum, over all executions, of the normalized time of this execution. In the rest of this paper, "time complexity" always refers to "worst-case time".

Contrary to popular belief, allowing link delays to vary between 0 and 1 does not mean that the worst case is achieved when link delays are exactly 1 , as this would preclude anomalous executions, in which some links are fast and some are slow. It is conceivable that the worst-case time performance either in communication or time occurs when the algorithm is "fooled" by such anomalous behavior. This is particularly true for evaluating comnunication complexity; for example, distributed BellmanFord algorithm when runs in asynchronous network requires exponential complexity; however if delays of all links are equal to 1 then number of messages is polynomial.

\subsubsection{Dynamic asynchronous network}

Dynamic asynchronous communication network is the main model considered in this paper. We make the standard assumptions [Fin79, Seg83, Gar89b] that failures/recoveries of links may cause loss of messages in transit, but are detected by the underlying data link protocol. We view the network topology as a part of the input. Input may have additional components, like link weights, etc.
We allow the input (topological and other) to change arbitrarily with time. The requirement imposed on a dynamic network protocol is to produce a correct final output once the input stabilizes. For example, the output of a dynamic shortest path algorithm will stabilize to shortest path on the final network topology with final edge weights.

Complexity measures for a dynamic network protocol refer to time and communication overhead introduced by a single (topology) change. Amortized Communication [Fin79, AAG87] is the number of messages sent by the protocol per input change, namely total number of messages sent divided by the number of changes. Quiescence Time [Fin79, AAG87] is the (normalized) time it takes to assimilate fully to a input (topology) change, i.e. the time from the last input change until the last cutput change.

\subsection{The Problems}

We consider here two inter-related tasks in dynamic asynchronous network, the one of constructing shortest paths, and the one of designing locp-free routing protocols.

\subsubsection{Constructing Shortest Paths}

The requirement imposed on dynamic network protocols, (shortest paths protocol in particular) is that once the topology of the network and the weights are stable, then eventually the correct output will be computed.

We consider the case of single source, and no negative cycles in the steady-state. For convenience, we look on the case of shortest paths of length (at most) $t$, where $t$ is a positive integer. We will want to express our upper bounds on time in terms of $t$, implicitly assuming $n \gg D \gg t$. We would like to be able to reconstruct shortest path of length $t$ in linear $(O(t))$ time after topological change investing only polynomial $(O(\operatorname{pol} y(n))$ number of messages per change.

\subsubsection{Loop-Free Routing Protocols}

The routing protocol consists of a dynamic shortest path protocol, in conjunction with certain forwarding protocol. Forwarding protocol uses the output of the shortest path protocol in order to deliver messages to thejr destinations. Typically, forwarding 
protocol is sending message to the parent of the node in the current shortest path tree.

As the shortest path protocol is updating the shortest paths trees, packets may enter into a (temporary) loop, in the sense that a packet enters the same node more than once. This phenomenon is called "looping". In some practical situations looping of packets is undesirable, and it is preferable that the protocol drops a packet, rather than allowing looping. Protocols that disallow looping are called loop-free routing protocols; such protocols have attracted a lot of attention in the literature.

\subsection{Existing work}

The problem has been considered extensively in the existing literature. We first survey the existing work in static networks, then survey work in dynamic networks, and finally outlinc our approach. In the rest of this section, we compare our approach with existing ones, and argue that it is superior to existing ones in terms of worst-case communication and time complexity.

\subsubsection{Static networks}

Purely asynchronous approach: is exemplified by the "most natural" distributed shortest path algorithm, called "Bellman-Ford" algorithm. This algorithm requires linear time in the sense that shortest paths of length $t$ are constructed in $O(t)$ time. However, in the worst case, it requires exponential (around $2^{n}$ ) messages. A particular example where this number of messages is sent is due to Gallager (private communication). There are two known approaches to overcome this problem.

Centralized synchronization approach: has been used in [Gal82, MS79]. The essence of this approach is that the algorithm synchronizes thru a central location. This enables to reduce the communication to polynomial, but makes convergence time very slow, approximately $O\left(n^{2}\right)$.

Local synchronization approach: This is the only known method to achieve (in static asynclıronous network) a linear tine slortest patiss algorithms with polynomial communication complexity. The essence of this approach is that we establish a synchronizer in the the network, which generates clock pulses that simulate the global clock. Second,

\begin{tabular}{|c|c|c|}
\hline Authors & Commun. & Time \\
\hline \hline [Taj77] & $2^{n}$ & $D$ \\
\hline [Gal82, MS79] & $t \cdot E$ & $t^{2}$ \\
\hline [Jaf80, Awe85] & $E \cdot t$ & $t$ \\
\hline \hline
\end{tabular}

Figure 1: Static Asynchronous Shortest Paths algorithms. Here, $t$ is the length of the shortest path.

we run synchronous shortest paths using the synchronizer. This approach has been suggested independently by Jaffe in 1980 [Jaf80] and by Awerbuch in 1984 [Awe85]. For our purpose, there is no need in using the sophisticated synchronizers of [Awe85], and the simplest synchronizers suffice. The summary of existing shortest paths algorithms in static asynchronous networks is given in Figure 1.

\subsubsection{Dynamic networks}

Special-purpose approach: the essence of this approach is attempting to design protocols that use specific properties of the shortest paths problem. This resulted in a series of very interesting protocols, that are ingeniously tailored for this problem.

The first sub-category here is that of somewhat "chaotic" algorithms, used in the old ARPANET algorithm [Taj77, MRR80]. Those algorithms have absolutely catastrophic performance, as they feature a a so-called "bouncing effect" [Gar89b, CRKG89]. The essence of this effect is that presence of outdated information (i.e. incorrect setting of initial conditions for the Bellman-Ford algorithm) "confuses" the protocol, and significantly slows down its convergence time.

The next sub-category is that of dynamic protocols built around static algorithms which use "centralized synchronization". Example of such approach is the celebrated Merlin-Segall protocol and its variations [MS79, SS81]. The importance of this work is that it yields for the first time shortest paths protocol that requires only polynomial communication and time. It convergence time is very slow, namely quadratic in the number $n$ of network nodes. Some researchers objected to such slow convergence, and looked after shortest paths algorithms that converge in linear time.

This triggered nexi sub-category of protocols, build around "purely asynchronous" static algorithms, e.g. [JM82, Hum81, Gar89b, Gar89a, 
CRKG89, RF89]. All algorithms in that category have linear $(O(n))$ time complexity, which constitutes an important improvement over [MS79] in terms of time. In some situations, some of those algorithms may have an edge over others; however, in the worst-case, all of those algorithms, even in the absence of topological changes, require exponential $\left(\Omega\left(2^{n}\right)\right)$ message complexity. This is not surprising, as they are based on static algorithms that are inherently exponential. (Whenever polynomial bounds are mentioned, they reflect the performance of the protocol in the best case, when link delays are synchronous; e.g., see [CRKG89, Gar89a].)

Global Reset approach: One may wonder why static synchronizers [Jaf80, Awe85] will not work in a dynamic environment. One reason for this is that the synchronizer cannot run as is in a network where edges may recover, since the endpoints of an edge that recovers can be far off from each other in their pulse numbers [A we85], namely, up to $n-1$.

As a remedy to the problem of making static protocols tolerant to topology changes, Finn [Fin79] introduced a concept of a Reset procedure. Roughly speaking, this is a way to "reset" the computation after a topological change. The essence of this technique is that after each topological change, the previous computations are destroyed and a new version of the protocol is started. Such Reset enables to adapt any static asynchronous protocol to dynamic asynchronous network. In particular, we can view the combination of a synchronizer and synchronous shortest paths algorithm as an asynchronous protocol, and reset this protocol for every topological change.

One problem with this approach is that the bounded-counters protocol of Finn [Fin79] is, in fact, incorrect, as shown by Soloway and Humblet [SH87]. Subsequently, the problem has been fixed by Soloway and Humblet [SH87]. The simplest and the most efficient reset procedure has been given by Afek, Awerbuch and Gafni in [AAG87].

There is another, more subtle problem. Since the destruction process starts at one place and must traverse the whole network, any locality properties of the original protocol are destroyed. As a result, the quiescence time (time until the network has fully assimilated the last topology change) of the resultant transformed protocol becomes as high as $\Omega(n)$, where $n$ is the number of network nodes. It is worth mentioning that one cannot claim better bounds, say, $O(D)$ time, where $D$ is the diameter, as the protocol of [AAG87] may in fact take $\Omega(n)$ time. This may be much greater than the running time of the original protocol. For example, if we are interested in reconstructing shortest paths of length $t \ll D \ll n$, this approach is quite inefficient.

The natural question here is whether suich a "global reset" can be performed locally, say, in $O(1)$ time. Observe that, no matter how local is the underlying computation, we cannot simulate it fast if we have separate clocks for each one of the $O(1)$ neighborhoods in the network, since this would have created congestion in the network.

\subsection{Our approach}

This paper overcomes the difficulties above as follows. The general idea is to achieve a direct simulation of the static synchronous network by the dynamic asynchronous network requiring only constant time overhead, and polynomial communication complexity. The heart of the simulation is a technique of [AS88], called a DYNAMIC SYNChroNIZER, which achieves "local" simulation of a global "clock" in a dynamic asynchronous network. The simulation protocol is based on an approach, perceiving "locality" as the key to fast adaptation to changes in network topology. This localization of operations will also prove efficient in handling situations in which the network is forever unstable. The major reason for this "localization" is due to the fact that we abandon Finn's blast-away method, where previous computations are destroyed. Instead, we take advantage of previous computations in order to speed up the response to a topological change.

Then, we run a static synchronous protocol on top of the dynamic synchronizer. This yields a shortest paths algorithm for dynamic networks which reconstructs shortest paths of length $t$ in $O(t)$ time, with only polynomial number of messages. This provides a positive answer to the question, posed by Cheng, Riley, Kumar and Garcia-Aceves-Luna [CRKC:89].

Comparison of our results with existing ones is given in the Figure 2.

\subsection{Structure of the rest of the paper}

In the rest of the paper, we proceed as follows. Section 2 first presents the solutions for shortest paths and loop-free routing problems in static synchronous networks, and then presents static syn- 


\begin{tabular}{|c|c|c|}
\hline Authors & Commun. & Time \\
\hline \hline [Taj77] & $2^{n}$ & $2^{n}$ \\
\hline$[$ MS79, SS81] & $n \cdot E$ & $t^{2}$ \\
\hline [JM82, Hum81] & $2^{n}$ & $n$ \\
\hline [CRKG89, Gar89b] & $2^{n}$ & $n$ \\
\hline [Awe85, AAG87] & $E \cdot t$ & $n+t$ \\
\hline This paper & $E \cdot t$ & $t$ \\
\hline \hline
\end{tabular}

Figure 2: Our Shortest Paths algorithm versus existing ones

chronizers which enable those protocols to run in static asynchronous networks. In Section 3, we present the dynamic synchronizers, which allow to run directly static synchronous protocols on dynamic asynchronous network.

\section{Static protocols}

\subsection{Shortest paths in static syn- chronous network}

The shortest path algorithm in synchronous network is quite simple. We assume an upper bound $t$ on length of the shortest path is known; the protocol can be easily modified to work for the case when $t$ is unknown.

Every node maintains a variable Parent ${ }_{p}$, which is a parent of the node in the pulse number $p$ of the protacol, and a variable Distance $(p, e)$, which is the distance values reported thru edge $e$ at pulse $p$, and is is an upper bound on the distance to the destination node if the next edge to be taken is $e$. At pulse $p$, Distance is set to be the minimum of Distance $(p-1, e)$, over all $e$. Observe that the value of Distance is monotonically decreasing with $p$.

In the following Figure 3 we give the code of Shortest-Paths stage for the static asynchronous network.

\subsection{Loop-free routing in static syn- chronous network}

Loop-free routing protocol is very easy to implement in a static synchronous network. This protocol forwards the message along the dynamic shortest path tree, without ever creating a loop. The idea is very simple: if message was created at pulse $p$, then the value of $p$ is appended to the message; it should

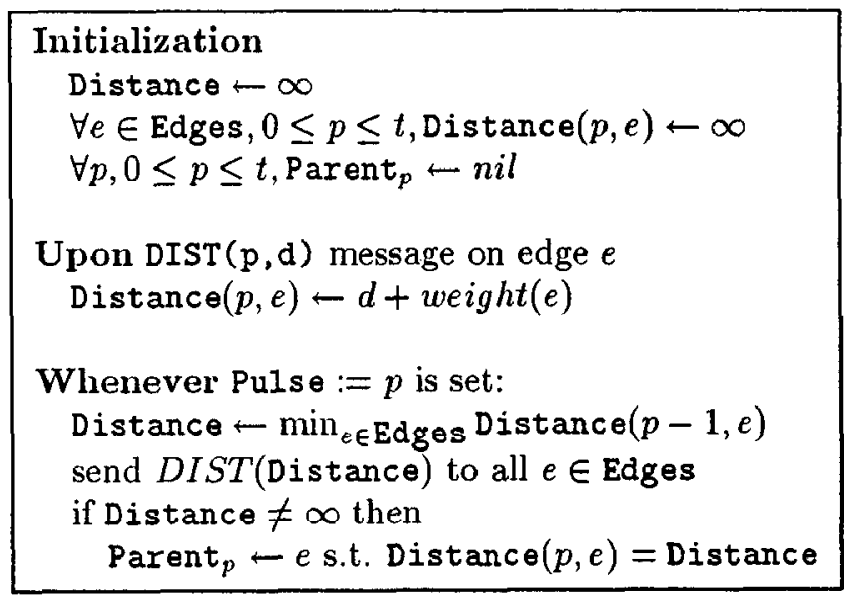

Figure 3: Bellman-Ford: node algorithm

\section{Initialization:}

message $M$ is generated while Pulse = p: Call Procedure FoRwaRD $(M, p)$

Upon arrival of message $(M, p)$ on $e$ if Pulse $(e) \geq p$ then

Call Procedure ForWaRd $(M, p-1)$ else drop packet $\quad / *$ inconsistency detected */

Procedure Forward $(M, p)$

if you are not the destination of $M$, then forward message $(M, p)$ to Parent $p$. else deliver message $M$ to host

Figure 4: Forwarding protocol: node algorithm

be forwarded by each node to Parent $t_{p}$, i.e. parent existing at pulse $p$. This way, message propagates along the tree existing at time $p$, thus never returning back to the same node.

In the following Figure 4 we give the code of Forwarding protocol for the static asynchronous network. The code contains a clause, that enables nodes to drop packets in case that "inconsistency" is detected. This clause will become relevant only later, when we will use this code in conjunction with other protocols in dynamic asynchronous network. 
Adjacent: the set of adjacent links

State: flag receiving values dropping,growing. The Synchronizer is operational only while State = growing.

Pulse: Highest pulse which was performed correctly until now.

Pulse(e): Estimate on Pulse of the node on the other end of edge $e$.

PULSE ( $p$ ) message carrying the parameter Pulse of the sender.

NEW-PulsE procedure that might start a new pulse.

Figure 5: Static synchronizer $\alpha$ : node variables and procedures

\subsection{Static synchronizer}

This protocol enables to emulate static synchronous network on a static asynchronous network. The protocol, first presented in [Awe85] is very simple: once all neighbors reported performing pulse $p-1$, the node performs pulse $p$.

The formal description of the synchronizer is given in Figures 5, 6. In order for the Static Synchronizer $\alpha$ to be operational, the variable State in this synchronizer should be initialized to growing. (The value of State is not changed by the static synchronizer itself.) 'I'he reason for introducing this variable into the code of the protocol is that in the future we will use Synchronizer $\alpha$ in the setting in which the value of State will be changed by some external protocol.

\section{Dynamic Synchronizers}

\subsection{Problem Statement}

\subsubsection{Preliminaries}

Following [AS88], in this section we define the notion of a dynamic synchronizer in such a way that it can be "reset" in a "local" way and is robust to topological changes.

The dynamic synchronizer has a parameter $t$, which represents the time complexity of some synchronous protocol. While the definition of the dynamic synchronizer seems to implicitly require

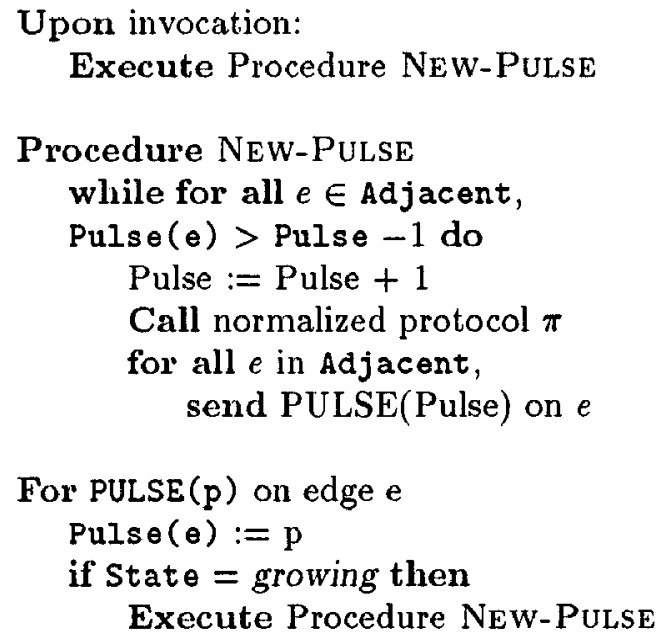

Figure 6: Static synchronizer $\alpha$ : node algorithm

knowledge $t$, in actual implementation we do not use this knowledge.

The dynamic synchronizer generates, as ar. output, a (finite) sequence of pulses at each node of the network. Each pulse is labeled with a pcsitive integer not exceeding $t$. The pulse labeled with $p$ is called pulse $p$. The pulse number at a node at a given time is the labeling of the last pulse generated at this node by that time, which represents the current "local time" at that node. Unlike "regular" time, "local time" is not necessarily monotonic, i.e. it may decrease as well as increase, similarly to "roll-back" mechanisms [KT87]. Thus, there may be many pulses at a node labeled with the same pulse number $p$.

Definition 3.1 A final pulse $p$ at a node is a pulse labeled $p$ such that all pulses following it are labeled by $p+1$ at least. A final message of pulse $p$ or edge $(u, w)$ is the message sent by $u$ to $w$ upon final pulse $p$ at $u$. The final pulses and messages must satisfy the specifications stated in Figure 7.

Example of sequence of pulses satisfying the conditions above is given in the following Figure 8.

\subsubsection{Complexity measures}

The complexity measures for dynamic synchronizers are defined as follows. Consider the set of executions of the dynamic synchronizer $\mathcal{S}$ in a dynamic 
Adaptivity: Final input change at a node precedes final pulse 1 .

Termination: Final pulse 1 is performed by each quiescent node.

Liveness: For all $1<p \leq t$, and for each quiescent node, if node receives final messages of pulse $p-1$ from all its quiescence neighbors, then it generates final pulse $p$.

Safety: For all $1 \leq p \leq t$, arrival of a final message of pulse $p$ at a node precedes final pulse $p+1$ at that node.

Figure 7: Specifications of a dynamic synchronizer

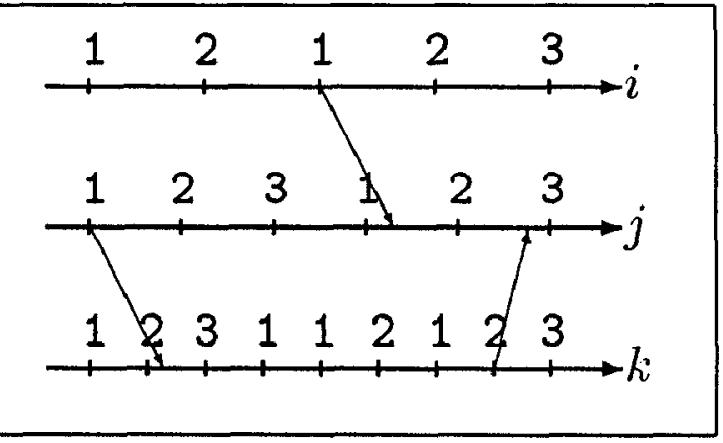

Figure 8: Timing diagram describing pulse sequences at nodes $i, j, k$ produced by a dynamic synchronizer. Arrows represent message sent from one node to another. The last three pulses at each node look "as if" generated by a static synchronizer. network, with $k$ input changes happening during the execution.

Notation 3.2 We introduce the following notations:

$\hat{C}_{\mathcal{S}}:$ is the Amortized Communication Complexity. This is the total number of messages sent, divided by $k \cdot t$.

$T_{i n i t}(\mathcal{S}):$ is the Initialization Time. This is the maximal time from the time a node reaches quiescence until it performs final pulse 1.

$T_{p u l s e}(\mathcal{S}):$ is the Pulse Time. This is the maximal difference between the time that all neighbors of a node have performed final pulse $p-1$ and the time a node performs final pulse $p$.

$\hat{T}_{\mathcal{S}}:$ is the Amortized Time Complexity. It is defined as $\hat{T}_{\mathcal{S}}=\frac{T_{\text {init }}(\mathcal{S})}{t}+T_{\text {pulse }}(\mathcal{S})+1$.

\subsection{Simple Synchronizer}

Following [AS88], a Dynamic synchronizer with $O(1)$ time can be easily achieved by a "naive" protocol, in which every node tries to raise its pulse number as high as possible, subject to the following two requirements:

1. The pulse number of a node can be increased to $p$ only after all neighbors increase their pulse number to $p-1$.

2. pulse number always drops to 0 after each input change

Observe that pulse drops must be performed to satisfy the Liveness condition. To implement 1), consider the following procedure, which will be referred to as the STATIC SYNCHRonizer $\alpha$; it is essentially identical to synchronizer $\alpha$ of [Awe85]. The essence of this procedure is that the pulse number of a node is increased to $p$ after all neighbors increase their pulse number to $p-1$. Note that knowledge of $t$ is not needed.

To implement 2), we add on top of the static synchronizer $\alpha$ a mechanism for dropping pulse numbers; this mechanism will be called Manager $\varepsilon$. Basically, it forces every node to drop its pulse number to 0 upon every input change, and report the drop in the pulse number to all neighbors. Upon receipt of this report, a neighbor with pulse number higher than 1 will drop its pulse number to 1 , and will 


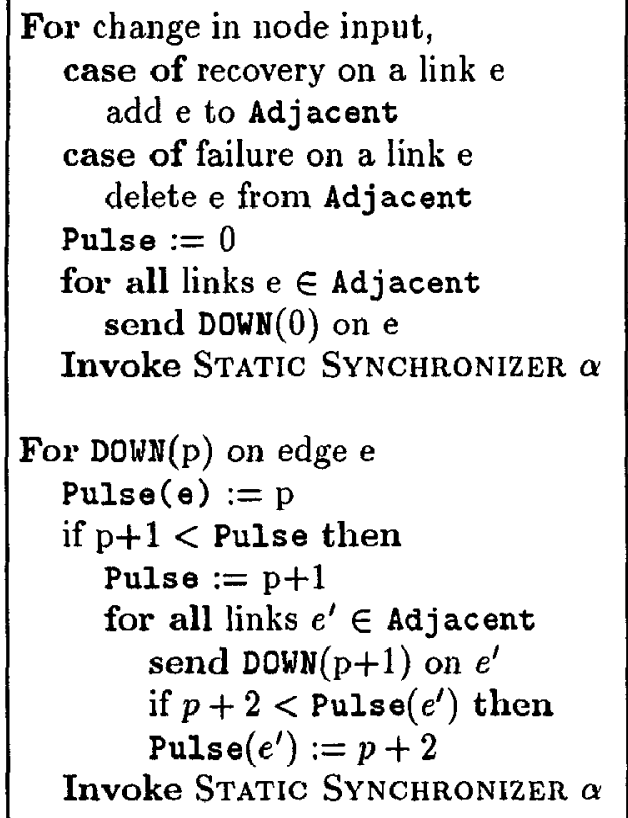

Figure 9: Manager $\varepsilon$

report this fact to all neighbors. When the latter report arrives at a node with pulse number higher than 2, it drops it pulse to 2, etc. After each pulse drop, a node executes static synchronizer $\alpha$ again, trying to increase its pulse number.

The code of Manager $\varepsilon$ is presented in Figure 9. The combination of Manager $\varepsilon$ together with static synchronizer $\alpha$ will be referred to as Dynamic Synchronizer $\varepsilon$.

Theorem 3.3 The dynamic synchronizer $\varepsilon$ indeed satisfies the Safety, Adaptivity, Termination, and Liveness properties of a dynamic synchronizer. Its time complexity is $\hat{T}_{\varepsilon}=O(1)$.

Comment: We do not claim any bounds on the communication complexity $\hat{C}_{\varepsilon}$. In the following subsection, we present a slightly more complex synchronizer, whose communication complexity is provably small, i.e. polynomial in the size of the network.

\subsection{Polynomial synchronizer}

\subsubsection{Outline}

The Final dynamic synchronizer, referred to as SYNchronizer $\zeta$, uses two subroutines. The first subroutine is the Static SYnchronizer $\alpha$, presented in the previous section. The second subroutine, called MaNager $\zeta$, can be viewed as a modification of Manager $\varepsilon$. The task of the Manager $\zeta$ is to suspend static synchronizer $\alpha$, delete all outdalied information by dropping the value of Pulse and then to resume static synchronizer $\alpha$, which will cause Pulse to increase.

It is essential for the communication complexity of the resulting protocol that, roughly speaking, all the pulse drops are performed before all pulse increases. In that respect, our strategy resembles the one used in [AAG87]. However, the implementation is somewhat different; it uses, among others, techniques of [DS80, AG87].

For simplicity, suppose first all the changes are performed "sequentially", i.e. long periods of time separate any two changes. A node which notices a change, originates a special "shock wave", which propagates thru the $t$-neighborhood of the origin of the wave, Every node at distance $1 \leq d \leq t$ of the origin will drop its pulse number to $d$, since all pulses from $d$ until $t$ have been performed with outdated information. Once the wave reaches the boundary of the $t$-neighborhood, it "reflects" back, as in [DS80]. Once all the "reflections" of the wave have been collected at the origin, it is clear that all the pulse numbers have been dropped. At this time, the origin starts propagating the "secondary" wave, which informs all the nodes in the $t$-neighborhood that they can start executing synchronizer $\alpha$, so that the pulse numbers will start increasing. In order to deterrnine the boundary of the $t$-neighborhood and the precise distance from the origin inside the $t$-neighborhood, we execute Breadth-First-Search (BFS) w.r.t. the origin. In the actual implementation, nodes do not need to know the value of $t$.

Now, if many changes happen in parallel, then each originator "conquers" all the nodes which are closer to it than to some other originator, effectively performing a Breadth First Search with respect to all the originators. During this process, one originator whose tree is growing "fast" may "conquer" nodes which should belong in fact to other ariginator; however the "true owner" will eventually retaliate, reconquering its nodes. The technique used in this paper to handle such problems is essentially a distributed version of Bellman-Ford algorithrn, in which nodes update their distances in a "greedy" manner, similarly to [Fre85, AG87]. We additionally impose a mechanism of acknowledgments [DS80] on top of this algorithm, so that origin can detect the 
top of this algorithm, so that origin can detect the time at which its tree stops growing.

\subsubsection{Detailed Description}

Every node keeps the following variables. Pulse describes number of the last pulse performed correctly. In-edge points to a neighbor which caused the most recent drop in the pulse number; the set of these pointers forms a forest of directed trees, rooted at the nodes which observed input changes. Each such tree will be called a drop tree. The variable State receives values dropping, growing; by changing this variable the MANAGER protocol resumes and suspends the STAtic Synchronizer $\alpha$.

Once a node with Pulse $>0$ notices a change in the input, it drops its Pulse to 0, sets In-edge to self and sets its State to dropping, thus suspending the operation of Static Synchronizer. Next, it executes procedure BroadCAST-Drop. In that procedure the node broadcasts a special DROP(Pulse) message over each adjacent edge $e$, setting $\operatorname{Ack}(\theta):=$ false.

Once a node receives a $\operatorname{DROP}(p)$ message over edge $e$, it acts as follows. If Pulse $\leq p+1$, then it sends back ACK $(\mathrm{p}+1)$ message. Otherwise, it sets Pulse := $p+1$, In-edge $:=e$, State $:=$ dropping and executes procedure BRoADCAST-DROP.

The meaning of the ACK message is that the subtree of the drop tree rooted at that node has stopped growing. Observe that the node may send many DROP messages while State $=$ dropping; however the pulse numbers in those messages are strictly decreasing. Thus, $\operatorname{ACK}(\mathrm{p})$ messages arriving at the node with $p>$ Pulse correspond to previous DROP message. Once an acknowledgment $A C K(p)$ arrives on edge $e$, the node acts as follows. If $p \neq$ Pulse, then this ACK is outdated and thus is discarded. Otherwise, if $p=\mathrm{Pulse}$, this is the ACK to the latest DROP message sent by the node. In the latter case, the node sets $A \operatorname{ck}(\theta):=$ true and executes procedure Converge.

In procedure Converge, the node cliecks whether $\operatorname{Ack}(e)=$ true for each adjacent link $e$. If so, the node will send ACK(Pulse-1) message on In-edge. Once the root of a drop tree detects that $\operatorname{Ack}(\theta)=$ true for all incident edges $e$, it decides that the "cleaning" process is over, and now is the time to resume the operation of synchronizer $\alpha$.

For that purpose, it executes the procedure BroadCast-Grow. In the procedure BroadCAST-
Grow, the node sends Grow message to all neighbors, and sets State := growing, thus resuming the operation of SYNCHRONIZER $\alpha$. Once a node receives GROW message on its In-edge, it executes procedure Broadcast-Grow.

The Manager $\zeta$ protocol is formally presented in Figures 10, 11. Figure 10 contains the declarations of the variables, messages and procedures used, and Figure 11 describes the node algorithm.

\subsubsection{Properties of dynamic synchronizer $\zeta$}

Theorem 3.4 The dynamic synchronizer $\zeta$ above indeed satisfies the Safety, Adaptivity, Termination, and Liveness properties of a dynamic synchronizer. Its complexities in communication and time are

$$
\begin{aligned}
& \hat{C}_{\zeta}=O(E) \\
& \hat{T}_{\zeta}=O(1)
\end{aligned}
$$

\subsection{Applications to Shortest Paths and Loop-Free Routing}

Combining either one of the "Manager" protocols in Figures 911 , respectively with the static synchronizer in Figure 6 and the shortest paths algorithm in Figure 3 yields a dynamic asynchronous shortest path algorithm. Adding the forwarding protocol in Figure 4, we get a dynamic asynchronous loop-free routing protocol.

\section{Summary}

In this paper, we argue that the best approach to achieve fast and message-efficient shortest paths algorithms in dynamic networks is via a direct simulation of the static synchronous network by the dynamic asynchronous network. This new approach yields a prolocol with linear lime and polynomial communication, thus answering positively the questions raised by Cheng, Riley, Kumar and GarciaAceves-Luna [CRKG89]. We believe that other problems in dynamic asynchronous networks can be tackled in a similar way.

\section{Acknowledgments}

Thanks are due to Jose Garcia-Aceves-Luna, Pierre Ilumblet, and George Varghese for helpful discussions. 
Variables joint with the synchronizer

Adjacent: the set of adjacent links.

State: flag, updated by the MANAGER protocol. Receives values dropping, growing. The SYNCHRONIZER is operational only while State = growing.

Pulse: Highest pulse which was performed correctly until now. Receives values $\{1,2, \ldots t-1, t\}$.

Pulse(e): Estimate on Pulse of the node on the other end of edge e. Receives values $\{1,2, \ldots t-$ $1, t\}$.

\section{Additional Variables}

$\operatorname{Ack}(\theta)$ : Binary flag, indicating whether an $A C K$ is expected on the edge $e$, maintained for all $e \in$ Adjacent.

In-edge: The parent of a node, maintained only while State = dropping.

\section{Messages}

$\operatorname{DROP}(p)$ : message carrying the parameter Pulse of the sender.

ACK $(p)$ : acknowledgment to DROP message.

\section{Procedures}

BROADCAST-DROP: procedure which broadcasts DROP messages.

BROADCAST-GRow: procedure which broadcasts GROW messages.

Converge: procedure which converges ACK messages towards roots.

Figure 10: Declarations of variables, messages, procedures
For change in node input

case recovery on a link e

Ack (e) := true

add e to Adjacent

case failure on a link $e$,

delete $e$ from Adjacent

if Pulse $\neq 0$ then

Execute Procedure PARENT-Switch

In-edge : $=$ nil

Pulse $:=0$

Execute Procedure Broadcast-Drop

Procedure PARENT-Switch

if In-edge $\neq$ nil then

send ACK (Pulse-1) on In-edge

Procedure BroadCAST-Drop

State := dropping

for all links $e \in$ Adjacent

send DROP (Pulse) on $e$

Ack $(e):=$ false

Execute Converge

For DROP $(p)$ on edge e

Pulse $(e):=p$

if $p+1 \geq$ Pulse then send $\operatorname{ACK}(p)$ on $e$ else

Execute Procedure PARENT-Switch

Pulse $:=p+1$

In-edge $:=e$

Execute Procedure BroAdcast-Drop

For ACK (p) message on edge $e$

if $p=$ Pulse then

Ack $(e):=$ true

Execute Procedure Convenae

Procedure Converge

if for all $e \in$ Adjacent, Ack $(e)=$ true then

if In-edge $\neq$ nil then send ACK (Pulse+1) on In-edge else Execute Procedure BRoADCAST-Grow

Procedure Broadcast-Grow

for all $e \in$ Adjacent send GROW on $e$

State := growing

For GROW message on edge $e$

if $e=$ In-edge then

Execute Procedure Broadcast-Grow

Figure 11: Dynamic synchronizer 


\section{References}

[AAG87] Yehuda Afek, Baruch Awerbuch, and Eli Gafni. Applying static network protocols to dynamic networks. In $28^{\text {th }}$ Annual Symposium on Foundations of Computer Science. IEEE, October 1987.

[AG87] Baruch Awerbuch and Robert G. Gallager. A new distributed algorithm to find breadth first search trees. IEEE Trans. on Info. Theory, IT33(3):315-322, May 1987.

[AS88] Baruch Awerbuch and Michael Sipser. Dynamic networks are as fast as static networks. In $29^{\text {th }}$ Annual Symposium on Foundations of Computer Science, pages 206-220. IEEE, October 1988.

[Awe85] Baruch Awerbuch. Complexity of network synchronization. $J$. of the $\Lambda C M, 32(4): 804$. 823, October 1985.

[CRKG89]

Chunhsiang Cheng, Ralph Riley, Srikanta P.R. Kumar, and Jose J. Garcia-Luna-Aceves. A loop-free extended Bellman-Ford routing protocol without bouncing effect. In Proc. of the ACM Symp. on Communication Architectures and Protocols, Austin, Texas, pages 224-236. ACM SIGCOMM, ACM, September 1989.

[DS80] Edsger W. Dijkstra and C. S. Scholten. Termination detection for diffusing computations. Info. Process. Letters, 11(1):1-4, August 1980.

[Fin79] Steven G. Finn. Resynch procedures and a fail-safe network protocol. IEEE Trans. on Commun., COM-27(6):840-845, June 1979.

[Fre85] Greg N. Frederickson. A single source shortest path algorithın for a planar distributed network. In Proceedings of 2 nd Symp. on Theoretical Aspects of Computer Science, January 1985.

[Gal82] Robert G. Gallager. Distributed minimum hop algorithms. Technical Report LIDS-P-1175, MIT, Lab. for Information and Decision Systems, January 1982.

[Gar89a] Jose J. Garcia-Luna-Aceves. Mininum hop routing algorithm based on distributed information. Computer Nctworks and ISDN, 16, 1989.

[Gar89b] Jose J. Garcia-Luna-Aceves. A unified approach to loop-free routing using distance vectors or link states. In Proc. of the ACM Symp. on Communication Architectures and Protocols, Austin, Texus, pages 212-223. ACN SIGCOMM, ACM, September 1989.
[Hum81] Pierre A. Humblet. An adaptive distributed dijkstra shortest path algorithm. Technical Report CICS-P-60, Center for Intelligent Control Systems, MIT, May 19881.

[Jaf80] Jeffrey Jaffe. Using signalling messages instead of clocks. Unpublished manuscript., 1980.

[JM82] J. Jaffe and F. Moss. A responsive distributed routing protocol. IEEE Trans. on Commun., COM-30(7, Part II):1758-1762, July 1982.

[KT87] R. Koo and S. Toueg. Checkpointing and rollback-recovery for distributed systems. IEEE Trans. on Software Eng., SE-13(1):2331, January 1987.

[MRR80] John McQuillan, Ira Richer, and Eric Rosen. The new routing algorithm for the arpanet. IEEE Trans. on Commun., 28(5):711-719, May 1980.

[MS79] P. Merlin and A. Segall. Failsafe distributed routing protocol. IEEE Trans. on Commun., COM-27:1280-1288, September 1979.

[RF89] Balasubramanian Rajagopalan and Michael Faiman. A new responsive distributed shortest path routing algorihtm. In Proc. of the ACM Symp. on Communication Architectures and Protocols, Austin, Texas, pages 237-246. ACM SIGCOMM, ACM, September 1989.

[Seg83] Adrian Segall. Distributed network protocols. IEEE Trans, on Info. Theory, IT-29(1):23-35, January 1983. Some details in technical report of same name, MIT Lab. for Info. and Decision Syst., LIDS-P-1015; Technion Dept. EE, Publ. 414, July 1981.

[SH187] Stuart R. Soloway and Pierre A. Humblet. On distributed network protocols for changing topologies. Technical Report LIDS-P-1564, MIT, Lab. for Information and Decision Systems, May 1987. Also have version from May 1986.

[SS81] A. Segall and M. Sidi. A failsafe distributed protocol for minimum delay routing. IEEE Trans. on Commun., COMf-29(5):689 695, May 1981.

[Taj77] W. P. Tajibnapis. A correctness proof of a topology information main tenance protocol for a distributed computer network. Comm. of the $A C M, 20(7): 477-485,1977$. 